\title{
AN EXPERIMENTAL STUDY OF LIH STOICHIOMETRY AT ELEVATED TEMPERATURES AND PRESSURES
}

\author{
A. J. Piwnskii \\ E. M. Lilley \\ G.S. Smith
}

June 25, 1975

Prepared ior U.S. Energy Research \& Development

Administration under contract No. W-7405-Eng-48

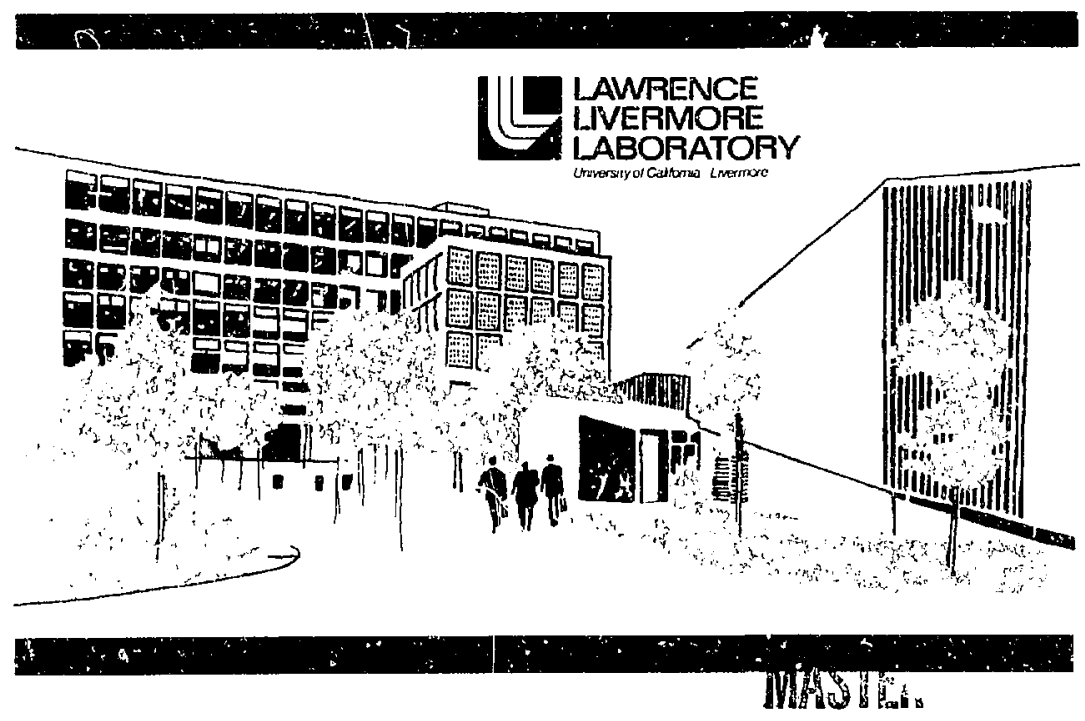




\section{NGIKI}

"l bos reporl twas prepare-d is an acculn! of avork spousated by the Unitus Sistes Goyermentent. Neither the Ungted States nor the United States litargy Restorch \& Devetopneml Administration, nom ally of their employes, nur any uf theit sulltiltars, subcoutracturs, of their implugers nakes dily wasranty, express or amplicd, ur assunies any letail tiability or responsibility for the aceutacs, completuness or usufulness of any infurmation. apparslus, product or proses so Jindersted, ar represents that its use wesuld not intrunge ptivaledy-owned rishts."

Printed in the United States of America Available from

National Technical Information Service

U.S. Department of Commerce 5285 Port Royal Road Springfield, Virginia 22151 Price: Printed Copy $\$$ :; Microfiche $\$ 2.25$

$$
\begin{gathered}
\text { "Pages } \\
\hline 1-50 \\
51-150 \\
151-325 \\
326-500 \\
501-1000
\end{gathered}
$$

N1'IS

Selling Price
$\$ 4.00$
$\$ 5.45$
$\$ 7.60$
$\$ 10.60$
$\$ 13.60$ 


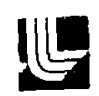

LAWRENCE LIVEFMORE LABORATORY

unversily ol Cabtomia Livermare.Cahrorna 94550

ICUL-31:50

\title{
AN EXPERIMENTAL STUDY OF LIH STOIC:AIOMETRY AT ELEVATED TEMPERATURES AND PRESSURES
}

\author{
A. I. ['in inskii

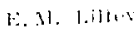 \\ (i.s. sin:? \\ hum 23,145
}

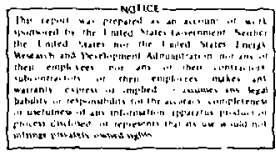




\title{
AN EXPERIMENTAL STUDY OF LIH STOICHIOMETRY AT ELEVATEI) TEMPERATURES AND PRESSURES
}

\begin{abstract}
We have investigated the stoichiometry that consisted of a Ab-Pd imner capsule and of Lith in the temperature interval 500 $900 \mathrm{~K}$ at hydrogen pressures between 2.5 and $6.0 \mathrm{GPa}$. The results and interpretation of these experimental data are complicated by the presence of ublquitous intermetalifc compounds from encapsulating materlals, which are formed at pressures below 4.0 GPa. The most definitive results of the cantalum outer capsule at a pressure of $5.0 \mathrm{GPa}$ and $733 \mathrm{~K}$. Thls run proviced no change in $L f H$ stolchlometry, nor any change In the Naci-type erystal structure, is a result of our investigation, we conclude that up to a pressure of $6.0 \mathrm{GPa}$ and a temperature of $803 \times$ ao quenchable Lili with $n$ > 1 can be produced.
\end{abstract} lavestigation were produced in an assembly

\section{Introduction}

Under suitable conditions elemental Lithlum reacts wlth hydrogen to form stolchionetric LfH. We have conducted a reconnalssance study to determine if LiH reacts further with hydrogen to form a quencisble nonstofchlometric $\mathrm{L}_{\mathbf{H}} \mathrm{n}_{\mathrm{n}}$, where $\mathrm{n}>1$. We have treated the ordinary hydride with hydiogen at pressures between 2.5 and 6.0 GPa In the remperature interval $500-900$ k. In addition to those uxperinisnts we have Invest1gated the efiect of time on $\mathrm{LiH}$ staichlometry under thuse conditlons. This report contalns the results of these experiments. All dats are reported in sl unlts.

\section{Experimental Procedures}

All experlments below 4,0 GPa were performed in a piocon-cylinder apparatus slmilar to that of Boyd and England. The high pressure die-and-sample assembly have been previously deacrlbed by Stephene ${ }^{2}$ and Stephens et a1. ${ }^{3}$ Figure 1 is a schematic lllustration of the die and Internal capsule arrangenent we employed in all our exper Iments. Temperature was measured using chromel-alimel thermocouples without pressure correction. Accuracy of all temperature readings $1 \mathrm{~s} \pm 20 \mathrm{~K}$. Pressures are accurate to $50.1 \mathrm{GPa}$.

The experiments conducted above 5.0 $\mathrm{GPa}$ were carried out in a 12.7-mm $\times 12,7-$ mm girdle anvil device previously described by Stromberg and Stephens ${ }^{4,5}$. In this apparatus, temperature was masured by 


\section{Top platen of piess}

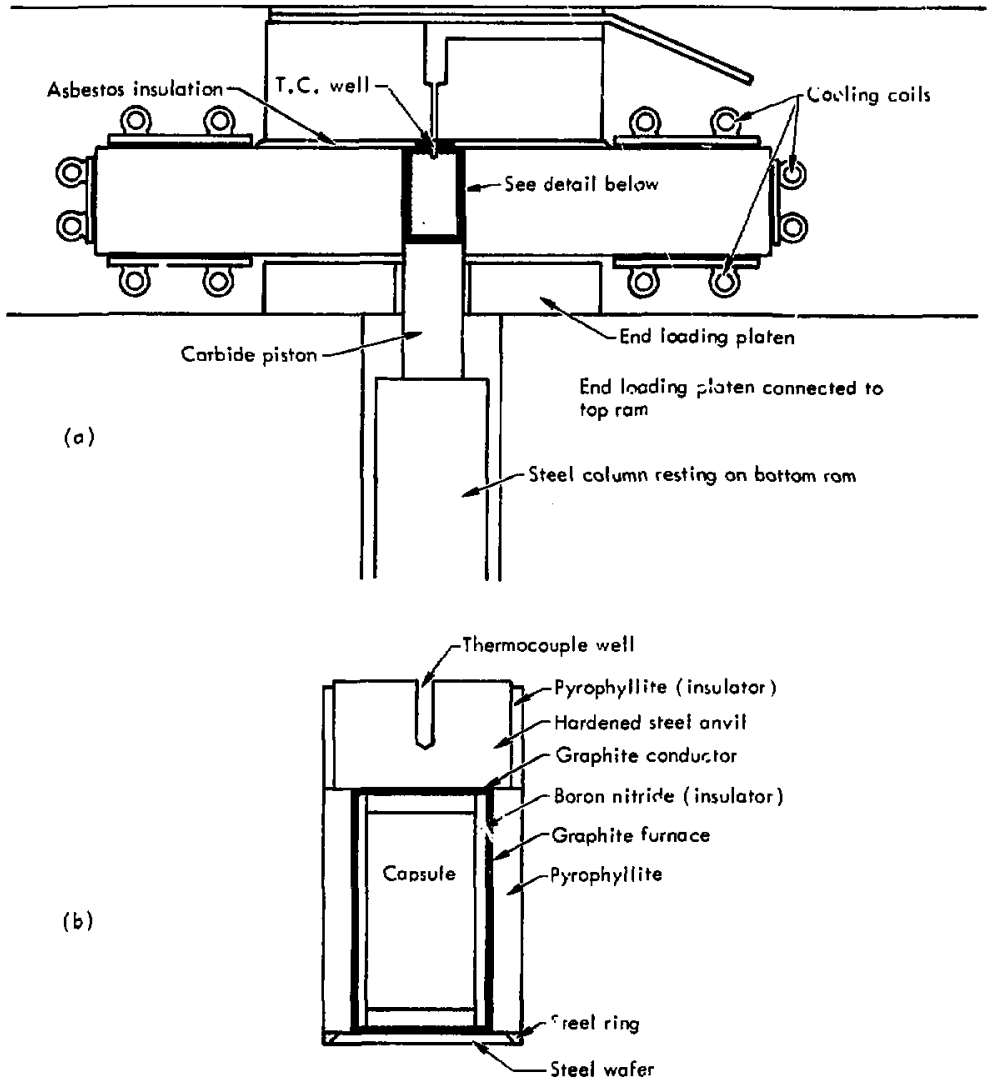

Fig, I High-pressure-die assembly in press, schemaric. (a) Overall view, (b) Detall of cupsule holder. 
moniloring wateage input which previously lad been callbrated by H. D. Stromberg as a function of temperature using a So-Mo 50 Ke therinocouple (see Fig. 2). wevacy of all readlings is $+25 \mathrm{k}$.

Hun produr:ts were analysed by $k$-ray powder diffaction techniques. The sample capsules were sawed open In an argonatwosplece glove box, The materlal was tren pulverlted and the samples sealed in thin-walled glass captllaries for use in Debye-Scherrer comeras. LiH gives a simple diffraction patcern of the $1 \mathrm{cc}$ NaCl type so that diffraction lines due to other materlals can be casily recognised. Because of fauliy seals Lioh impurittes wert observed on rare occastons in some diffrartion patcerns. These patterns were excluded from further consideration.

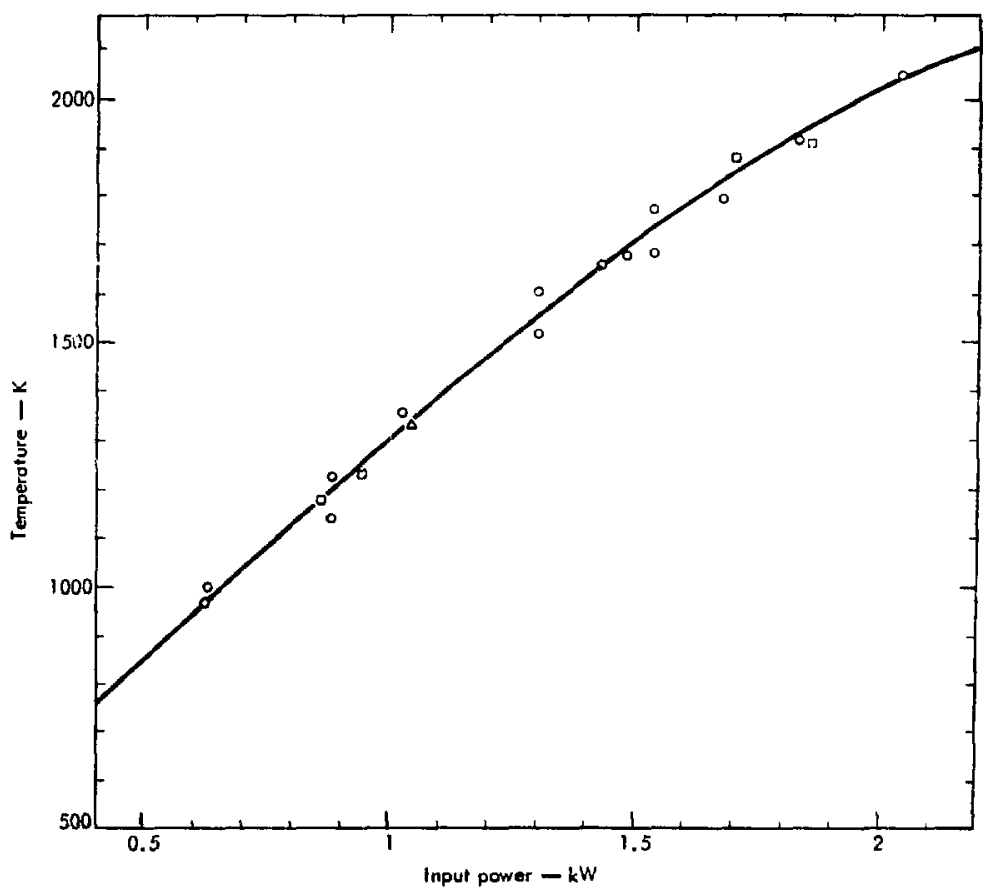

Fig. 2 Girdle tamperature calibration uging Mo-No 50 Re thermocouple. See Ref. 6. 
Xiray difiraction annlyas were curried out by mariual or compater searches si the Joint Comite toe for Powder Diffracfion standardy inorganic t1le conststag of approximatels 15,000 standard pilterrs. Some of the run products were allalysed tor metal impurities atther by emission spectrosiopy or x-ray fluerescence techis ques.

\section{Sample Preparation and Contaimment}

Uise of the most vexing and complex dspects of the high pressure flivestigation was the fabrleat lon oi sultable capsule strangerants and cormulation of experimental Druedures to achieve successful runs. becalse of the reactive nature of the material:i employed, as well as the multipliity of problems encountered, we simall discuss our efiorts in this area in detall.

The early experiments were performed ucllizing an lncernul gold capsule whose geometry is schematicaliy lilugtrated in Fig. 3. Powdered LIH wis concatned within an Armas Iran Inner capsule. This was surrounded by powdered $\mathrm{BeH}_{2}$, and boch sumponents wite contalined $\mathbf{n}$ a seaied gold capsule. This arrangement will be called Design 1. This, as well as guccegolvo capsule desigus, occuples the "capsule" cavicy lllustrated in Fig. Ib.

Ihe thesis underlying these early experiments was that, at yuffictently high

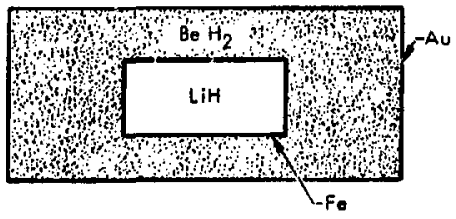

Fig. 3 Capsule design 1. temperatures, the Boll. - He $+\mathrm{H}_{2}$ reaction would occur in the oster capsule? The hydrugen would then permeate through the steel liner and react with tite $L$ fil. This expartiner at arrangement was only partally successtul, Hydrogen gas was indeed liberated ac elevated cemperaturey; however, the thin gold outer copsule way too weak to sontain the extreme internal presisurets.

Design 2, an attwapt to remedy this situation, is sciteontically fllustrited in $7 \mathrm{ig}$. 4. The big change is tie nature of the outer gold capsule. In Design 2, il Is a sold capsule 15.9 wide with a 3.18-im whll thickness, whereas in lDesign 1 wall thlckness was only $0.76 \mathrm{~mm}$, in

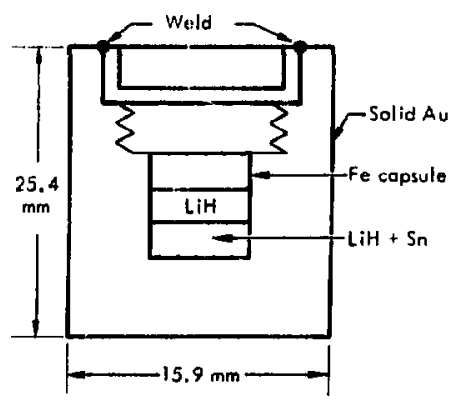

Fig. 4 Capsule degign 2. 
idditlon, Desifu 2 wat different in that 1: genurated hydropen by reaction of a mLxlure of mutallic th and l.th luaded in the outer cupsule.

Jesign 2 was structurally sound and wist used In severil runs, Contaminution of clie produces by lithlum-gold Intertoetellit compunds was a serious Irawback, lisw:ver.

The appearance of this contaminant Mlase prompled further madifications, with dustentele stainless stesl, Armco 21-6-9

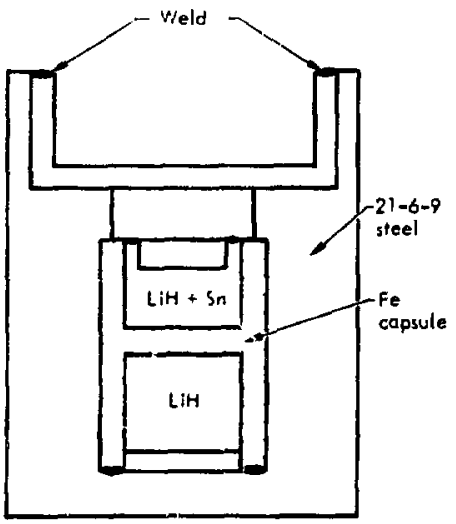

Fig. 5 Cansule design 3.
(21Cr-6N1-9Mn), replacing the solid gold outer capisule. Thls steel is much sirunger than gold and recent tests reveal extreme resistance to iydrogen embritclement ${ }^{-1 C}$. Chemical analysis of the material tested by Frlik et al. is proviced in labja 1 . Dosign 3 is given in Fig. 5. Destgn 3 ivas successfully employed In several experiments; it deterred bold-lithium intermetallic compound formation and provided hign strength to prevent capsule rupture.

Table 1. Composition of Armco 21-6-9 (in element we \%).

\begin{tabular}{|c|c|c|}
\hline & Bar & Plate \\
\hline C & 0.013 & 0.03 \\
\hline Si & 0.11 & 0.37 \\
\hline $\mathrm{Mn}$ & 8.86 & 8.88 \\
\hline NI & 6.06 & 6,62 \\
\hline$C \mathbf{r}$ & 19.7 & 20.03 \\
\hline Mo & $-m-$ & --- \\
\hline $\mathbf{P}$ & 0.022 & 0.020 \\
\hline $\mathbf{S}$ & 0.011 & 0.013 \\
\hline $\mathrm{Cu}$ & 0.19 & 0.23 \\
\hline $\mathbf{N}$ & 0.29 & 0.33 \\
\hline $\mathrm{Fe}$ & Bat-nce & balanes \\
\hline
\end{tabular}

${ }^{a}$ Data from Frick et al." 


\section{Experimental Results}

Table 2 15ts results of all experiwents that, in oir opinion, produced hydrogen under conditions of the run. h-ray diffraction resules appear in table 3.

Runs 4 and 5 lasted tor only a matter of minutes and were aborted because of electrical shorts in the high pressure system. This prablem was overcome in Run 6 by using onica and asbestos paper between the die and back-up block and by placing cooling coils around the die assembly (see Fig. 1). Typical die taidperatures encountared in longer runs using the couling colls are portrayed in Fig. 6 . which plots die cemperature as a function of time for Run 6 .

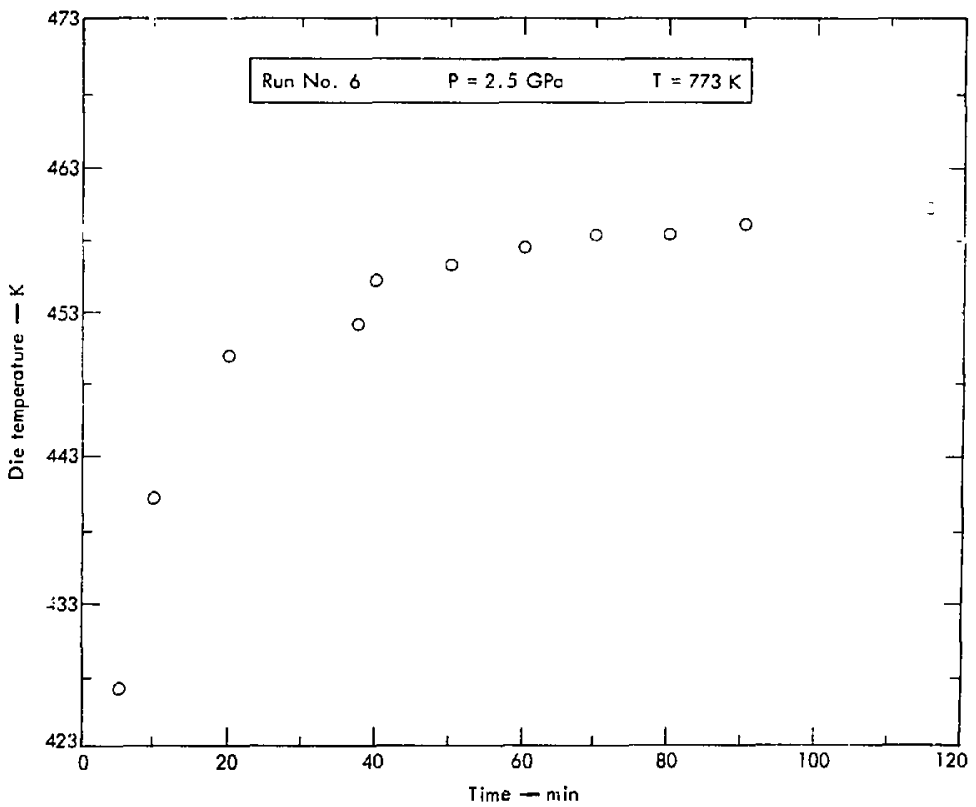

Fig. 6 Die temperature as a function of run duration for experiment No. 6. 
lable 2, Experimental kuns on :ir.

\begin{tabular}{|c|c|c|c|c|c|c|c|c|c|c|}
\hline $\begin{array}{l}h ; n \\
x, .\end{array}$ & $\begin{array}{c}\text { Pretsis } \\
\text { whts }\end{array}$ & 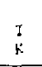 & $\begin{array}{l}\text { :ime } \\
\text { in }\end{array}$ & $\begin{array}{l}\text { jEstesig } \\
\text { =aterial }\end{array}$ & 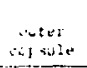 & 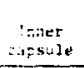 & 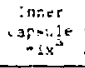 & $\begin{array}{l}\text { Sdr:le } \\
\Delta e+i n d\end{array}$ & 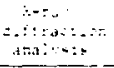 & $\therefore-=E=$ \\
\hline$i$ & $\therefore 3$ & 575 & U.25 & $\begin{array}{l}\text { Powduted } \\
\text { lith }\end{array}$ & 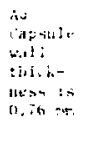 & 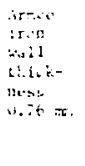 & $\therefore s+s+$ & : & & 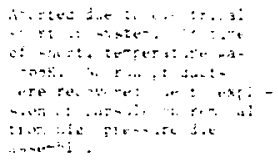 \\
\hline 5 & $\therefore 3$ & $i 7$ & 0.067 & $\begin{array}{l}\text { bithiertud } \\
\text { l.1!l }\end{array}$ & 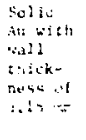 & 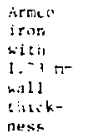 & $1.111+\sin$ & - & $\therefore$ & 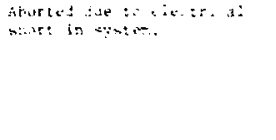 \\
\hline b & $\therefore 5$ & $\because ;$ & $\ldots 1$ & 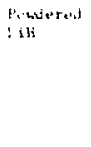 & $\begin{array}{l}\text { will } \\
\text { sud }\end{array}$ & $\begin{array}{l}\text { irran." } \\
\text { in ant }\end{array}$ & $\therefore 1+\infty+2$ & : & & 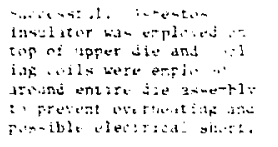 \\
\hline 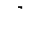 & 1.) & $n+1$ & U..1 & $\begin{array}{l}\text { lowdered } \\
\text { i. } \mathrm{H}\end{array}$ & $\begin{array}{l}\text { :olifi } \\
\text { As }\end{array}$ & tron & $|\leq|:-\div \leq 12$ & 2 & $x$ & 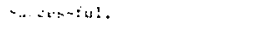 \\
\hline 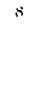 & $\therefore \dot{3}$ & $\because: \vdots$ & 0.1. & $\begin{array}{l}\text { interdertd } \\
\text { lit }\end{array}$ & $\begin{array}{l}\text { bolli. } \\
\text { su }\end{array}$ & 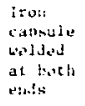 & i.i.t+sin & 2 & $\therefore$ & anstid! \\
\hline-1 & $\therefore 4$ & $\because$ & $\therefore 1 \mu^{n}$ & $\begin{array}{l}\text { lithidud } \\
\text { litis }\end{array}$ & $\begin{array}{l}\therefore+2 i d \\
\text { stsit } \\
\text { atiut } \\
21-n-9\end{array}$ & 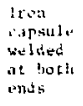 & 1. $14+5 i 1$ & 3 & $x$ & 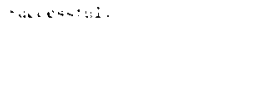 \\
\hline w & $\therefore j$ & $7 ?+$ & $\therefore 0$ & $\begin{array}{l}\text { rressed } \\
\text { pellet } \\
\because \quad \text { ill }\end{array}$ & $\begin{array}{l}\text { stcol } \\
\text { ailoy } \\
21-b-9\end{array}$ & $\begin{array}{l}\text { Iron } \\
\text { citpsule } \\
\text { belded } \\
\text { at both } \\
\text { eids }\end{array}$ & $\begin{array}{l}\text { fressidil } \\
\text { peidut } \\
\text { of } \\
\text { l.ilitsin }\end{array}$ & , & $\because$ & :ivi:ast:al. \\
\hline 11 & 2.5 & $5 \%$ & .14 & $\begin{array}{l}\text { Presied } \\
\text { Fetitet } \\
\text { ot } 1.14\end{array}$ & $\begin{array}{l}\text { Steel } \\
\text { al buy } \\
21-b-9\end{array}$ & $\begin{array}{l}\text { Jron } \\
\text { eapsinl: } \\
\text { holded } \\
\text { at botll } \\
\text { ends }\end{array}$ & $\begin{array}{l}\text { Prasisod } \\
\text { pelle } \\
\text { of } \\
\text { l.iH+sin }\end{array}$ & 1 & & 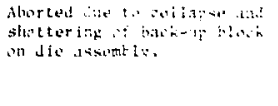 \\
\hline 12 & 2.5 & 773 & 7.83 & $\begin{array}{l}\text { Prosised } \\
\text { pollet } \\
\text { ni l, lil }\end{array}$ & $\begin{array}{l}\text { SLet } \\
1110 y \\
31-6-9\end{array}$ & $\begin{array}{l}\text { Iron } \\
\text { capsult } \\
\text { welded } \\
\text { at both } \\
\text { ends }\end{array}$ & $\begin{array}{l}\text { Preased } \\
\text { pellet } \\
\text { o[ } \\
\text { l. }[1]+5 i n\end{array}$ & 3 & $x$ & Sutenesion ul. \\
\hline
\end{tabular}


Table 2. (cont'd)

\begin{tabular}{|c|c|c|c|c|c|c|c|c|c|c|}
\hline $\begin{array}{l}\text { Run } \\
\text { ro. }\end{array}$ & $\begin{array}{c}\text { press. } \\
\text { GPa }\end{array}$ & $\begin{array}{l}y \\
k\end{array}$ & $\begin{array}{c}\text { Time } \\
\text { tir }\end{array}$ & $\begin{array}{l}\text { Starting } \\
\text { macertal }\end{array}$ & $\begin{array}{l}\text { Outer } \\
\text { capsule }\end{array}$ & $\begin{array}{c}\text { Inner } \\
\text { capsule }\end{array}$ & $\begin{array}{l}\text { Inner } \\
\text { capsule } \\
\text { mixa }\end{array}$ & $\begin{array}{l}\text { Sample } \\
\text { design }\end{array}$ & $\begin{array}{l}\text { X-ray } \\
\text { diffraction } \\
\text { analyalo }\end{array}$ & Conments \\
\hline 11 & 2.6 & 773 & 2.0 & $\begin{array}{l}\text { Presged } \\
\text { pellat } \\
\text { of LiH }\end{array}$ & $\begin{array}{l}\text { Steel } \\
\text { alloy } \\
21-6-9\end{array}$ & $\begin{array}{l}\text { Nickel } \\
\text { capsule }\end{array}$ & $\begin{array}{l}\text { Pressed } \\
\text { pellet } \\
\text { of } \\
\text { LiH+Sn }\end{array}$ & 3 & $x$ & Succesaful. \\
\hline 14 & 2.4 & 773 & 0.25 & $\begin{array}{l}\text { Pressgd } \\
\text { pellet } \\
\text { of Lit }\end{array}$ & $\begin{array}{l}\text { Steel } \\
\text { aIloy } \\
21-6-9\end{array}$ & $\mathrm{Ag}-\mathrm{Pd}$ & $\begin{array}{l}\text { Pressed } \\
\text { pellet } \\
\text { of } \\
\text { LIH+Sn }\end{array}$ & 3 & $\mathrm{X}$ & $\begin{array}{l}\text { Successful, but } 21-6-9 \\
\text { extremely ambrittled, } \mathrm{Ag}-\mathrm{Pd} \\
\text { changed color to bronze. }\end{array}$ \\
\hline I5 & 5.0 & 773 & 0,50 & $\begin{array}{l}\text { Pellet } \\
\text { of LIH }\end{array}$ & TantaIum & $\begin{array}{l}\text { Plat inum } \\
\text { inner } \\
\text { capsule } \\
\text { unwelded }\end{array}$ & $\mathrm{LiH}+\mathrm{Sn}$ & 4 & $\mathrm{x}$ & Succegsful. \\
\hline Ló & 0.0 & 803 & 0.16 & $\begin{array}{l}\text { Pellet } \\
\text { of } 1 \mathrm{H}\end{array}$ & Tantalum & $\begin{array}{l}\text { Platinum } \\
\text { Inner } \\
\text { capsule } \\
\text { unvelded }\end{array}$ & $\mathrm{Lx} H+\mathrm{Sn}$ & 4 & $\mathrm{x}$ & Successful. \\
\hline 17 & 5.0 & 273 & 0.50 & $\begin{array}{l}\text { lellet } \\
\text { of } \mathrm{LIH}\end{array}$ & Tantalum & $\begin{array}{l}\text { Ag-Pd } \\
\text { inner } \\
\text { capsule }\end{array}$ & $\mathrm{LIH+Sn}$ & 4 & $\mathrm{x}$ & Successfu1. \\
\hline
\end{tabular}

In the case of girdle-anvil runs 15,16 , and $17, \mathrm{~L}, \mathrm{H}+\mathrm{Sn} 1 \mathrm{~s}$ the outer capgule mix. There was no inner mix used in.sample design 4.

Runs 6,7 , and 8 were completed successfully using Design 2. Run products For these experiments, which werc performed at 2.5 and $3.5 \mathrm{GPa}$, were complicated by the presence of large quantities of goldlithium Intermetallic compounda (see Fig. 3). In particular, the $\mathrm{Au}_{3} \mathrm{LI}$ is of the $\mathrm{Cu}_{3}$ Au structure type ${ }^{L 1}$ with $a=396,8 \mathrm{pm}$. The strung contaminant lines overshadowed several weak diffraction lines which we could not identify. Table 3 11sts the unknown present in Runs 6, 7, and 8 as phase $X$.
Run 9 was undertaken to test the feasibility of using Armco 21-6-9 as capsule material at elevated temperatura and preasures in an aitempt to suppress the formation of gold-lithium intermetalif compounds. The components of the experiment consisted of a

- omall inner mild-oteel capsule welded at both enda to provide a closed syster,

- a large mass of $\mathrm{LIH}+\mathrm{Sn}$ surrounding the mild steel capsule, and

- a large outer capsule composed of the 21-6-9 alloy. 
Table 3. Debye - Scherrer x-ray powder ifffraction results of experimental runu on LiH.

\begin{tabular}{|c|c|c|c|}
\hline Run No. & State of $\mathrm{Lth}$ & Other phases present & Comment:: \\
\hline 5 & $\begin{array}{l}\text { Normal, } \\
\text { stoichiometric LiH }\end{array}$ & $\begin{array}{l}\text { Strong amounts of probably } \\
\text { AujLi in addition to small } \\
\text { quautities of a gold-rich } \\
\text { gold-ilthium solution. } \\
\text { Weak dif Eraction lines of } \\
\text { unknown phase } x \text {. }\end{array}$ & $\begin{array}{l}\text { The stoichiometric LiH is } \\
\text { present in small amounts. The } \\
\text { Aujli is of the Cu }{ }^{A u} \text { struc- } \\
\text { ture typa with a }=397.1 \text { pmi } \\
\text { the terminal Au-Li solid } \\
\text { solution has a }=396.8 \mathrm{pm} \text {. } \\
\text { See Ref. } 11 \text {. }\end{array}$ \\
\hline 7 & & $\begin{array}{l}\text { Essentfally identical to } \\
\text { Run } 5\end{array}$ & \\
\hline 8 & & $\begin{array}{l}\text { Essentially Identical to } \\
\text { Run } 5\end{array}$ & \\
\hline 9 & $\begin{array}{l}\text { Normal, } \\
\text { stolchiometric LiH }\end{array}$ & $\begin{array}{l}\text { Stainless steel and SiC. } \\
\text { Au-L: intermetallic com- } \\
\text { pounds are ahsent. }\end{array}$ & $\begin{array}{l}\text { New stee and carbide impuri- } \\
\text { ties probably introduced when } \\
\text { capsule was mechanically } \\
\text { opened. }\end{array}$ \\
\hline 10 & $\begin{array}{l}\text { Normal, } \\
\text { stolehiometric LiH }\end{array}$ & $\begin{array}{l}\text { Sta1nless steel and SiC. } \\
\text { Au-L1 compounds are absent. } \\
\text { Diffraction lines of new } \\
\text { phase Y. Emission spec- } \\
\text { troscopy shows there heavy } \\
\text { metal inpurities; SR>3 wt.\% } \\
\text { Fe } 0.3 \text { wt. \%; Ni } 0.3 \text { wt.\%. }\end{array}$ & $\begin{array}{l}\text { Phase } Y \text { diffraction lines are } \\
\text { not found in a reference } 11 \text { brary } \\
\text { containing approximately } 15,000 \\
\text { Facterns. } \\
\text { Approximately } 50 \% \text { of unknown } \\
\text { lines are accounted for in a } \\
\text { rough manner by } \mathrm{Li}_{3} \mathrm{Na}_{3} \mathrm{Co}_{2} \mathrm{~F}_{12} \text {. } \\
\text { Phase } \mathrm{Y} \text { could possibly contain } \\
\text { soue } \mathrm{Li}_{3} \mathrm{Fe}_{2}\left(\mathrm{~L}_{1} \mathrm{H}_{4}\right)_{3} \text { or } \\
\mathrm{Li}_{3} \mathrm{Sn}_{2}\left(\mathrm{l}_{4} \mathrm{HH}_{4}\right)_{3} \text {. }\end{array}$ \\
\hline 12 & $\begin{array}{l}\text { Normal, } \\
\text { otolehiometric } \mathrm{LiH}\end{array}$ & $\begin{array}{l}\text { New set of weak diffraction } \\
\text { lines, phase } Z \text {, distinct } \\
\text { from fcc LiH and from } \\
\text { phase Y lines. Endssion } \\
\text { spectroscopy shows: } \\
\text { Sn } \geq 2 \text { ut.\%, Fe } \geq 0.4 \text { wt. } \% \text {. }\end{array}$ & $\begin{array}{l}\text { New phase } z \text { lines correspond } \\
\text { to no presently known structure. }\end{array}$ \\
\hline 13 & $\begin{array}{l}\text { Normal, } \\
\text { sto ikfometric LiH }\end{array}$ & $\begin{array}{l}\text { Austenite or } \mathrm{Ni}_{\mathrm{x}} \mathrm{C}, \mathrm{x}>4 \\
\text { and strong amounts of unknown } \\
\text { phage, Fluorescence ana- } \\
\text { lysis shows: Ni strong: } \\
\mathrm{Ca}, \mathrm{Fe} \text {, As, } \mathrm{Zr} \text { trace. }\end{array}$ & $\begin{array}{l}\text { Strong amounts of new unknown } \\
\text { phase or phases; product is } \\
\text { dark gray. }\end{array}$ \\
\hline 14 & $\begin{array}{l}\text { Doubtful normal } \\
\text { L1H }\end{array}$ & $\begin{array}{l}\text { Lines of unknown phase. } \\
\text { Eluorescence analysis } \\
\text { shows: Major; Pd, Ag less } \\
\text { strong, Minor: Pt, N1, Zr, } \\
\text { Mo. }\end{array}$ & $\begin{array}{l}\text { Very complex diferaction } \\
\text { pattern. Product i.s dark in } \\
\text { color. }\end{array}$ \\
\hline
\end{tabular}




\begin{tabular}{|c|c|c|c|}
\hline Run No. & State of LiH & Other phases present & Comments \\
\hline 15 & $\begin{array}{l}\text { Normal, } \\
\text { stoichionetric LiH }\end{array}$ & $\begin{array}{l}\text { Other unknown phase; } \\
\text { Fluorescence analysis ohows: } \\
\text { Major; Pt, Zn, MInor: Sr, } \\
\angle \mathrm{r} \text {. Fe. }\end{array}$ & $\begin{array}{l}\text { Very complex diffraction } \\
\text { pattern. Strong amount of } \\
\text { normal LiH. Produet is light. }\end{array}$ \\
\hline 16 & $\begin{array}{l}\text { Normal, } \\
\text { stoichiometrlc LAH }\end{array}$ & Lines due to $\mathrm{Pt}$ and $\mathrm{Ta}$. & $\begin{array}{l}\text { Relatively simple and "cl tan" } \\
\text { patterr; all difEraction lines } \\
\text { accounted. Strong LiH present. }\end{array}$ \\
\hline 17 & $\begin{array}{l}\text { Nortali, } \\
\text { stolchiometric LiH }\end{array}$ & $\begin{array}{l}\text { Primitive cubic phase } \\
\text { almost certainly AgPd } 3 \text {. } \\
\text { Fluorescence analysls shows: } \\
\text { Ag, Pd strong; } 2 \text { n less } \\
\text { strong. }\end{array}$ & $\begin{array}{l}\text { Relatively simple and "clean" } \\
\text { pattern. Strong amounts of } \\
\text { normal LiH and weak amounts of } \\
\text { primitive cublc phase with } \\
\text { lattice constant }=393 \text { pm. } \\
\text { Product is light in color. }\end{array}$ \\
\hline
\end{tabular}

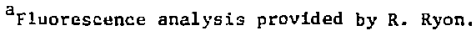

Daca reported in Table 2 indicate that tha capsule survived $10 \mathrm{~m} 1 \mathrm{n}$ at. $2.5 \mathrm{GPa}$ and $773 \mathrm{k}$. X-ray Diffraction analysis (see rable 3) detected no gold-lithlum 1atermetallic compounds.

Encouraged by the performance of the 21-6-9 allo*, we made rurs 10 and 12 for 2 and 7.83 hours, respectively, using Design 3 in crder to assess the effect of time at elevated cemperature and pressure on the stofctiometry of LIH. Both runs produced extremely complicated results (see Table 3). Run 10 contalned a new set of diffraction 1lies, which we are presently terming phase $Y$. Running a computer search ut1lizing a reference library containing approximately 15,000 known patterns resulted in no unequivocal ident1fication of Phase $Y$. Approximately half of the unknown lines are accounted for in a general way by $\mathrm{LI}_{3} \mathrm{Na}_{3} \mathrm{Co}_{2} \mathrm{~F}_{12}$, a flourlde garnet. Run 12 (see Table 3) produced another new set of diffraction 1ines. This set, much weaker In intensity than thos characteristic of Fhase $Y$, are being called Phase 2 . We a:e curratly unable to account for the Phase $z$ lines in terms of any known structure. A spectrochemical analya1s of runs 10 and 12 appears In Table 4.

Because of the unknown phases present 1n runs 10 and 12, we decided to modify the inner capsule material and assess the utflity of nickel and As-Pd. Rung 13 and 14 were performed at the $773 \mathrm{~K}$ 1 sotherm and 2.6 and $2.4 \mathrm{GPa}$, respectively, using nickel and Ag-Pd. Data presented In Table 3 reveal that both experiments produced very complicated $x$-ray diffraction patterns, in addition to normal LiH. Apparent $1 y$, substitution of nickei and Ag-Pd for the 21-6-9 alloy did not supress the formation of contaminant phases.

In view of the fallure to produce uncontaminated run products at pressures between 2.4 and $3.5 \mathrm{GPa}$, we dectded to 
Table 4. Spectrochemtial analyses of LiH runs no, 10 and 12 a $^{\text {a }}$

\begin{tabular}{|c|c|c|c|}
\hline $\begin{array}{l}\text { Analysis: } \\
\qquad \mathrm{Sn}\end{array}$ & $\begin{array}{c}10 \\
>30,000\end{array}$ & $\begin{array}{c}12 \\
\$ 20,000\end{array}$ & $\begin{array}{l}\text { The following elements were not detected in } \\
\text { efther sample: }\end{array}$ \\
\hline Fe & 3,000 & 4,000 & $4000 \mathrm{~K}$ \\
\hline $\mathrm{Ni}$ & 3,000 & 100 & $.400 \mathrm{Ns}, \mathrm{Hg}$ \\
\hline in & 900 & 200 & <100 Cd, se, Na, P, Sb, Th, zn \\
\hline $\mathrm{Cr}$ & 150 & 200 & $-40 \quad N b$ \\
\hline 51 & 200 & 150 & $<10 \mathrm{Ba}, \mathrm{Ge}, \mathrm{Mo}, \mathrm{Pb}, \mathrm{Tl}$ \\
\hline B & 150 & 100 & $<4 \quad \mathrm{Co}, \mathrm{In}, \mathrm{V}, \mathrm{Zr}$ \\
\hline $\mathrm{Cu}$ & 150 & 70 & \\
\hline $\mathrm{Ca}$ & 30 & 40 & \\
\hline Al & 30 & 20 & \\
\hline$B I$ & 15 & nd $<10^{b}$ & \\
\hline$A g$ & $<3$ & 10 & \\
\hline $\mathrm{Be}$ & $\geqslant 9$ & nd $<4$ & \\
\hline Mg & 6 & 7 & \\
\hline $\mathrm{Sr}$ & 3 & $<4$ & \\
\hline
\end{tabular}

"Values are approximate ppm by weight for the Impurity elements Iisted.

$b_{\text {nd }}$ - not detected.

Analyst: E. Peck

perfurm a series of runs at presgures between 5.0 and $6.0 \mathrm{GPa}$. Runs 15,16 , and 17 were carried out in a glrdle anvil device using capsule design 4 (see Fig. 7). Tantalum served as the outer capsule material; platinum and $\mathrm{AB}-\mathrm{Pd}$ were the Inner capoule components. Results of these experiments are IIsted in Tables 2 and 3. Run $15(5.0 \mathrm{GPa} / 773 \mathrm{~K})$ Frotuced a variety of unknown phases with complicaced $x-r a y$ diffraction patterns, Runs 16 and 17 were successful; they produced matertals that ylelded oimple and "clean" patterns. Run 16 (6.0 GPa/803 K) product gave a strong normal L1H pattern with amall amounts of $\mathrm{T}_{\mathrm{L}}$ and $\mathrm{Ta}$ as contaninants. Run 17 (5.0 GPa/773 K) product also gave strong Indication of normal $L f H$. However, 1t contained traces of what seems to be a primitive cubic phase, almost certalnly $\mathrm{AgPd}_{3}$, possessing a lattice constant of $393 \mathrm{pm} . \quad \mathrm{X}-\mathrm{ray}$ fliorescence analysis indicated that Run 17 contained small amounts of zine.

Thus, we nee that of all the experimenta performed, the least contaminated was Run 17. Table 3 shows that it contalned no other form of Lith than the normal. 


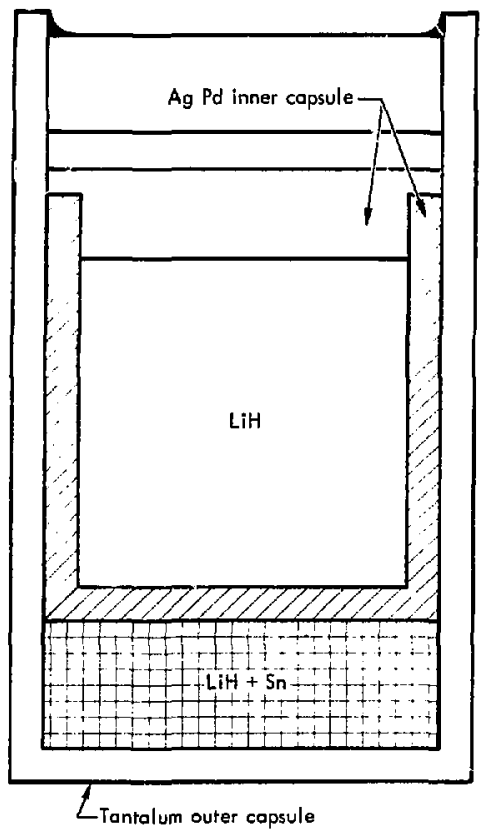

Fig. 7 Capsule design 4. 


\section{Discussion}

We have Investigated the stolihiometry of $L$ it in the temperature interval 500 $900 \mathrm{~K}$ between 2.5 and $6.0 \mathrm{GPa}$. The result $\mathrm{B}$ and Interpretation of these experimental data are complicaled by the nature and multiplicity of contatalnating fntermetallic compounds formed at pressures lower than $4.0 \mathrm{CPa}$.

The nost definfive results of the investigation were produced in an assembly that congisted of a AB-Pd inner capsule and tantalum outer capsule at a pressure of $5.0 \mathrm{GPa}$ and $773 \mathrm{~K}$. This run produced no change in $21 H$ stoichiometry, nor any change in the NaCl-type crystal structure. As a result of our investigation, we conclude that up to a pressure of $6.0 \mathrm{GPa}$ and a temperature of $803 \mathrm{~K}$ no quenchable LiH $_{n}$ with $\mathrm{n}>1$ occurs.
It 1s well known that many compounds possessing the rock salt structure transform into the CsCl structure at elevated pressures. Enploying two aiverse potential functions, Schumacher ${ }^{12}$ calculated that lif would undergo a phase cransicton between 0.305 and $0.380 \mathrm{GPa}$. His calculation 1s predicated on the fact that $\mathrm{LHH}$ is an tonic solid. Our resulta do not verify schumacher's theoretical predictlons; at least such a phase cannot be retained at ambient temperatures and pressures. Olinger and Halleck ${ }^{13}$ recently found that Lit "retalas the low pressure NaCl structure to $12 \mathrm{GPa}$ " However, Johnson ${ }^{14}$ mentioned that indirect evidence from shock wave experiments euggested the possibility of a transfomation at presaures greater than $12 \mathrm{GPa}$. If this transition is confirmed, it still remains to be deternined whether $\mathrm{LH}$ possessing, the isC1 structure couid exhibft nonstoichiometry.

\section{Acknowledgements}

We thank Q. Johnson, H, Leider, $H$. D. Stromberg, and $H$. Weed for useful discussions, H. Dye:, F. Silve and c. Griffiths for assistance, B. Bonner,
Q. Jolunson, A. Luba, H. Lefder, and H.D. Stromberg for a review of the manuscript . 


\section{References}

1. F. Boyd and J. England, "Development of High-Preesure Apparatus," in Annual Report of Director of Geophyaical Laboratory, Yearbook 57 (1958) pp. 170-173.

2. D. Stephens, J. Geophyg, Reg, 69 (14), 2967 (1964).

3. D. Stephens, E. Lilley, and H. Louls, Jutern, J. Rock Mech. MIn. Sci. I, 257 (1970).

4. H. D. Stromberg and D. Stephens, Bul1. Am. Ceram. Soc. 49 (12), 1030 (1970).

5. H. D. Stromberg and D. Stephens, Process for Produc1ng Sincered Diamond Compact and Products, U.3. Patent 3,574,580 (1971).

6. H. D. Stromberg, Lawrence L1vermore Laboratory, umpubl1shed results (1966).

7. W. Mueller, J. Blackledge, and G. Llbowltz, Metal Hydrides (Academic Press, New York, 1968) PP. 235 and 554.

8. V. Frick, G. Janser, und J. Brown, "Enhanced Flawgrowth in SSE Main Engine Alloys in High Pressure Gaseous Hydrogen," in Space Smuttle Materials, vol. 3 (Society of Aerospace Naterfils and Process Engineers, 1971) pp. 567-604."

9. H. Gray, Hydrogen Environment Embrittlement, NASA Technology Memorandum TMX-68088 (1972).

10. R. Vandervoort, Metals Eng. Quart. 2, 10 (1972).

11. W. Pearson, A Handbook of Lattlce Spacing ind Structures of Metals and Al Loys, vol. 2 (Pergamon Press, 1967).

12. D. Schumacher, Phys. Rev. 126 (5), 1679 (1962).

$\therefore$. B. Olinger and P. Halleck, App. Phys, Lett. 24, 536 (1974).

14. Q. C. Johnson, Lawrence Livermore Laboratory, private commulcation (1974). 